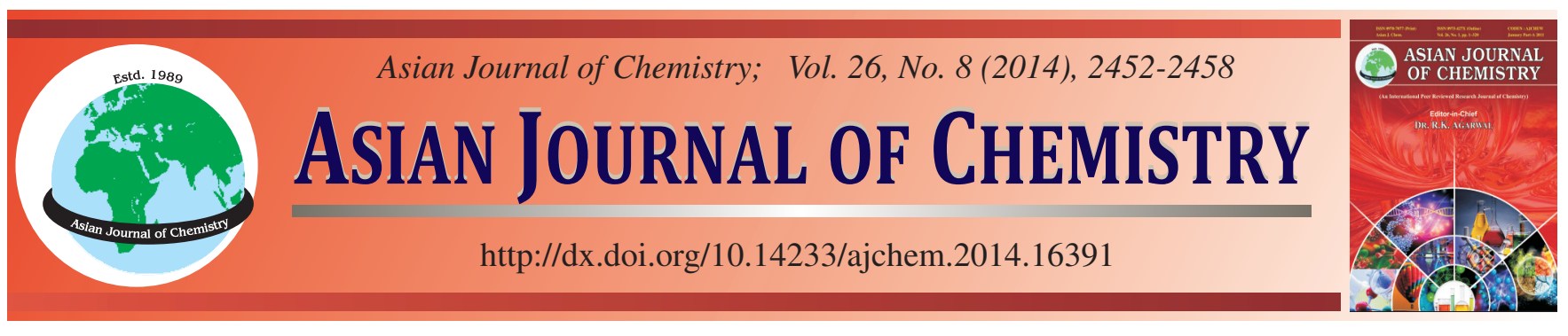

\title{
Extraction and Quantification of Lornoxicam in Human Plasma by Liquid Chromatography-Tandem Mass Spectrometry in Positive Ion Mode
}

\section{Nalini Kanta Sahoo ${ }^{1, *}$, Madhusmita Sahu ${ }^{1}$, Podilapu Srinivasa Rao ${ }^{1}$ and Goutam Ghosh ${ }^{2}$}

${ }^{1}$ Yalamarty Pharmacy College, Tarluwada, Visakhapatnam-530 052, India

${ }^{2}$ School of Pharmaceutical Sciences, Siksha 'O' Anusandhan University, Bhubaneswar-751 003, India

*Corresponding author: E-mail: sahoo.nalini@gmail.com

\begin{abstract}
A novel liquid chromatography tandem mass spectrometry method is described for the quantitative determination of lornoxicam in human $\mathrm{K}_{2}$ EDTA plasma in positive ion mode and validated using piroxicam as internal standard according to linearity, selectivity, precision, recovery and various stability studies. Sample preparation was accomplished by liquid liquid extraction technique. The eluted samples were chromatographed on ACE C18 $(150 \times 4.6 \mathrm{~mm}, 5 \mu)$ column (agilent technologies) using a mobile phase consisting of HPLC grade acetonitrile: $0.3 \%$ formic acid buffer $(80: 20 \mathrm{v} / \mathrm{v})$ with injection volume of $15 \mu \mathrm{L}$ and a run time of $3 \mathrm{~min}$. The precursor to product ion transitions $\mathrm{m} / \mathrm{z}, 372.10$ to 121.10 (lornoxicam) and $\mathrm{m} / \mathrm{z}, 332.10$ to 95.20 (piroxicam, IS) were used for quantization. The calibration graph of lornoxicam was linear with $\mathrm{r}^{2}>0.990$ over a concentration range of $5.086 \mathrm{ng} / \mathrm{mL}$ to $1518.325 \mathrm{ng} / \mathrm{mL}$. CV \% of intra- and inter-day precisions were found satisfactory and well within the limits. The drug was found to be stable for the studied parameters and found to be interference free for matrix effect with appreciable recovery. The novelty of the method makes it highly valuable, rapid, selective and sencitive for quantification of lornoxicam in human plasma and can be used in therapeutic drug monitoring of this drug.
\end{abstract}

Keywords: Lornoxicam, High-performance liquid chromatography, Mass spectrometry, Human plasma.

\section{INTRODUCTION}

Lornoxicam((3E)-6-chloro-3-[hydroxy (pyridin-2ylamino)methylene]-2-methyl-2,3-dihydro-4H-thieno[2,3e] $[1,2]$ thiazin-4-one 1,1-dioxide) is a non-steroidal antiinflammatory drug (NSAID) ${ }^{1}$. Lornoxicam is a compound in the same chemical class as piroxicam, meloxicam and tenoxicam with potent antiinflammatory, antipyretic and analgesic activity. Lornoxicam (chlortenoxicam), is a new nonsteroidal antiinflammatory drug (NSAID) of oxicam class. It is distinguished from established oxicams by a relatively short elimination halflife, lornoxicam inhibits the COX-1/COX-2 system, the production of interleukin- 6 and the inducible NO synthase ${ }^{2}$. It may be applied by the intramuscular or intravenous route; its bioavailability after oral application is approximately $90 \%$. Although its elimination half-life is only about $4 \mathrm{~h}$, the duration of effect is approximately $8 \mathrm{~h}$, analogous to other acidic antipyretic analgesics. The analgesic potency of lornoxicam is remarkable. In doses of $16 \mathrm{mg}$ (i.m.) its analgesic effect is comparable with that of $20 \mathrm{mg}$ morphine (i.m.) or $50 \mathrm{mg}$ tramadol (i.v. $)^{3}$. It acts by nonselective inhibition of cyclooxygenase- 1 and 2. It is prescribed for osteoarthritis, rheumatoid arthritis, acute lumbar sciatica conditions and postoperative pain management ${ }^{4}$. In the literatures a voltammetric ${ }^{5}$, polarograhic $^{6}$, UV spectrophotmetric ${ }^{7}, \mathrm{LC} / \mathrm{MS} / \mathrm{MS}^{8,9}, \mathrm{HPTLC}^{10}$ and high performance liquid chromatographic (HPLC) ${ }^{11-13}$ methods were reported for the analysis of lornoxicam. Due to the increasing importance of speed and reliability of analysis in bioanalytical laboratories, a new method for determination of lornoxicam in human plasma with a short time of analysis (3 min) is described in this work. The LC-MS/MS technique was successfully employed to provide a satisfactory sensitivity and selectivity in a desirable time of chromatographic run.

\section{EXPERIMENTAL}

Lornoxicam (Fig. 1) was purchased from Cirex Pharmaceuticals Limited, India and Piroxicam, the internal standard (Fig. 2), from Mankind Pharma Limited (Calcutta, India). HPLC grade Acetonitrile and methanol were obtained from J.T. Baker. HPLC grade water was procured from Rankem pharma. Formic acid, ethyl acetate and ammonia solution (HPLC grade) were obtained from MERCK.

The liquid chromatographic system consist of LC Shimadzu LC10 from Shimadzu, an auto sampler of Shimadzu (SIL-HTc) coupled with an applied Bio systems SCIEX a triple quadrupole mass spectrometer (API 4000) with electro spray ionization 
<smiles>CN1/C(=C(\O)Nc2ccccn2)C(=O)c2sc(Cl)cc2S1(=O)=O</smiles>

Fig. 1. Chemical structure of lornoxicam<smiles>CN1C(C(=O)Nc2ccccn2)=C(O)c2ccccc2S1(=O)=O</smiles>

Fig. 2. Chemical structure of piroxicam

(ESI) used for analysis. Date of acquisition and processing were controlled by applied bio systems/MDS SCIEX analyst software (version 1.4.2) with ACE 3 C18 column $(150 \times 4.6$ $\mathrm{mm}, 5 \mu)$.

The chromatographic analysis was performed by using a mobile phase of HPLC grade acetonitrile: $0.3 \%$ formic acid buffer $(80: 20 \mathrm{v} / \mathrm{v})$ with flow rate $1 \mathrm{~mL} / \mathrm{min}$ by positive ion mode (API 4000). Detection is performed by atmospheric pressure electro spray ionization (ESI) tandem mass spectrometry in positive ion mode. The chromatograms were recorded. Detection of the ions were performed by multiple reaction monitoring (MRM) of the transitions $\mathrm{m} / \mathrm{z}, 372.10$ and 121.10 for lornoxicam and $m / z, 332.10$ and 95.20 for the internal standard.

Preparation of stock standard and working solutions of lornoxicam: The stock solution of lornoxicam was prepared by dissolving $5 \mathrm{mg}$ of lornoxicam in $0.25 \%$ ammonia solution in methanol and made up the volume with the same in a $5 \mathrm{~mL}$ volumetric flask to produce a solution of $1000000 \mathrm{ng} / \mathrm{mL}$. This solution was kept in refrigerator at $2-8^{\circ} \mathrm{C}$. The stock solutions were diluted to suitable concentrations using diluent for spiking into plasma to obtain calibration curve (CC) standards, quality control (QC) samples for further use. All other dilutions were made in mobile phase.

Preparation of stock solution of piroxicam (internal standard): A stock solution of internal standard (IS) was prepared by dissolving $5 \mathrm{mg}$ of piroxicam in diluent (mixture of HPLC grade acetonitrile and water in a ratio (60:40) and made up the volume with the same in a $5 \mathrm{~mL}$ volumetric flask to produce a solution of $1000000 \mathrm{ng} / \mathrm{mL}$. This solution was kept in refrigerator at $2-8{ }^{\circ} \mathrm{C}$. Working internal standard solutions were prepared by suitably diluting the above mentioned stock solution afresh before use.

Preparation of calibration curve standards and quality control (QC) samples: Calibration curve standard consisting of a set of nine non-zero concentrations ranging from 5.086 to $1518.325 \mathrm{ng} / \mathrm{mL}$ of lornoxicam was prepared. Prepared quality control samples consisted of concentrations of 5.123 $\mathrm{ng} / \mathrm{mL}$ (lower limit of quantification quality control sample), $15.069 \mathrm{ng} / \mathrm{mL}$ (lower quality control sample), $251.148 \mathrm{ng} / \mathrm{mL}$ (middle quality control sample-1), $751.940 \mathrm{ng} / \mathrm{mL}$ (middle quality control sample-2) and $1303.189 \mathrm{ng} / \mathrm{mL}$ (higher quality control sample) for lornoxicam. These samples were stored at $-70 \pm 10{ }^{\circ} \mathrm{C}$ until use. Twelve sets of LQC and HQC samples were stored at deep freezer to check stability.

Sample preparation procedure: After bulk spiking, aliquots of $200 \mu \mathrm{L}$ for CCs and $200 \mu \mathrm{L}$ for QCs of spiked plasma samples were pipetted out into a prelabelled polypropylene micro centrifuge tubes and then all the bulk spiked samples were stored to deep freezer at $-70 \pm 10{ }^{\circ} \mathrm{C}$, except twelve replicates each of LQC and HQC, which were stored in deep freezer for generation of stability data. The thawed samples were vortexed to ensure complete mixing of the contents. 100 $\mu \mathrm{L}$ of the plasma sample was pipetted into stoppered test tubes, $20 \mu \mathrm{L}(2012.760 \mu \mathrm{g} / \mathrm{mL}$ of piroxicam) internal standard spiking solution were added to it and vortexed, except in blank plasma samples where $20 \mu \mathrm{L}$ diluent was added to it and vortexed. Then $25 \mu \mathrm{L}$ of $10 \%$ formic acid buffer was added to it and vortexed. Followed by addition of $5 \mathrm{~mL}$ of ethyl acetate and shaken for $20 \mathrm{~min}$ on reciprocating shaker at $200 \mathrm{rpm}$. Samples were centrifuged at $4000 \mathrm{rpm}$ for $5 \mathrm{~min}$ at $4{ }^{\circ} \mathrm{C}$. Then supernatant organic layer $(4 \mathrm{~mL})$ was transferred to prelabelled glass dry test tubes and evaporated to dryness in turbovap at $40{ }^{\circ} \mathrm{C}$. The samples were reconstituted in $1000 \mu \mathrm{L}$ of mobile phase and $15 \mu \mathrm{L}$ sample was injected to HPLC with MS-MS detection.

Method validation: The method was validated for selectivity, linearity, accuracy, precision, recovery, stability and carry over test according to the principles of the FDA industry guidance.

Sensitivity: The lowest limit of reliable quantification for lornoxicam was set at the concentration of the LLOQ i.e., 5.071 $\mathrm{ng} / \mathrm{mL}$. The precision and accuracy for lornoxicam at this concentration was estimated.

Linearity: The linearity of calibration curve for lornoxicam was assessed at nine concentration levels in the range of 5.086 $\mathrm{ng} / \mathrm{mL}$ to $1518.325 \mathrm{ng} / \mathrm{mL}$ in plasma samples. Peak area ratios for each solution against its corresponding concentration were measured and the calibration curve was obtained from the least squares linear regression presented with their correlation coefficient.

Extraction recovery: Twenty four blank matrix samples were processed and six sets of each blanks samples were reconstituted with the aqueous QC dilutions at low, middle and high concentration without internal standard, which represents $100 \%$ extraction of analyte(s) (non-extracted samples). Six blanks were reconstituted with the internal standard solution, which represents $100 \%$ extraction of internal standard. (Nonextracted sample). The non-extracted samples were injected. The recovery comparison samples of lornoxicam were compared against extracted samples of LQC, MQC2 and HQC of PA BATCH-I (precission and accuracy). The recovery comparison samples of internal standard were compared against the response of internal standard in MQC2 level. 
$\mathrm{R}(\%)=($ Psbe $/$ Psae $) \times 100$

where: $\mathrm{R}$ is extraction recovery, Psbe is the mean value of the peak area responses obtained from plasma samples spiked with analyte before extraction and Psae is the mean value of the peak area responses obtained from plasma samples spiked with analyte after extraction.

Accuracy and precision: Intra assay precision and accuracy were determined by analyzing six replicates at five different QC levels in two runs on the same day. Inter-assay precision and accuracy were determined by analyzing six replicates at five different QC levels on five different runs. The acceptance criteria included accuracy within $=15 \%$ deviation (SD) from the nominal values, except LLOQ QC, where it should be $\leq 20 \%$ and a precision of $\leq 15 \%$ relative standard deviation (RSD), except for LLOQ QC, where it should be $\leq$ $20 \%$.

Stability: Stability of lornoxicam in plasma was performed using six replicates of two QC samples at low and high levels. Samples were prepared by spiking drug-free plasma with appropriate volumes of standard solutions of lornoxicam. The stability was evaluated with different studies such as room temperature stock solution stability, refrigerated stock solution stability, room temperature spiking solution stability, refrigerated spiking solution stability, freeze-thaw, short term stability, bench top stability etc. Stability tests were conducted to evaluate the analyte stability in stock solutions and in plasma samples under different conditions. The stock solution stability at room temperature and refrigerated conditions $\left(2-8^{\circ} \mathrm{C}\right)$ was performed by comparing the area response of the analytes (stability samples) with the response of the sample prepared from fresh stock solution. Bench top stability $(6 \mathrm{~h})$, processed sample stability (auto sampler stability for $32 \mathrm{~h}$ ), freeze thaw stability (four cycles), reinjection stability ( $24 \mathrm{~h}$ ), wet extract stability (30 h) and plasma samples stability at $-20^{\circ} \mathrm{C}$ were performed at $\mathrm{LQC}$ and HQC levels using six replicates at each level. Samples were considered to be stable if assay values were within the acceptable limits of accuracy $(\leq 15 \% \mathrm{SD})$ and precision $(\leq 15 \%$ RSD).

Matrix effect test of lornoxicam: Two sets of extracted blank plasma samples each containing six tubes (plasma taken from six different lots) are taken. One set of tubes are reconstituted with equivalent aqueous concentration of LQC and the other set of tubes are reconstituted with equivalent aqueous concentration of HQC. These samples are known as post spiked samples. These samples are analyzed along with equivalent aqueous LQC and HQC samples. The matrix effect is evaluated by determining the $\%$ response ratio using the formula.

$\%$ Re sponse ratio $=\frac{\text { Mean area ratio of post spiked samples }}{\text { Mean area ratio of equivalent aqueous samples }} \times 100$

\section{RESULTS AND DISCUSSION}

The main aim of this work was to develop a rapid and selective analytical method including an efficient and reproducible sample clean-up step for quantitative analysis of lornoxicam in human plasma. Subsequently, a simple and inexpensive extraction procedure that could be implemented in monitoring laboratories provided an assay well suited for real time analysis. In optimizing the chromatographic conditions, the formic acid solution was adopted in the mobile phase of the HPLC in order to suppress the tailing phenomena of chromatographic peaks of lornoxicam and piroxicam. Besides ethyl acetate buffer was investigated and the inclusion made the chromatographic peaks sharp and symmetric. Further experimental results showed that acidifying the mobile phase with formic acid also contributed to improve peak shapes of lornoxicam and piroxicam. Therefore, a concentration of $0.3 \%$ formic acid was used in mobile phase. The acceptable retention and separation of lornoxicam and piroxicam was obtained by using an elution system of acetonitrile: $0.3 \%$ formic acid $80: 20, \mathrm{v} / \mathrm{v})$ as the mobile phase. The LC/MS/ MS method described here satisfies the requirement of routine analyses since it has a short run time ( $3 \mathrm{~min}$ ), which has advantages over other methods described in the literature. The MS optimization was performed by direct injection of lornoxicam and piroxicam into the mass spectrometer. The mass parameters were optimized to obtain better ionization of lornoxicam and piroxicam molecules. The full scan spectrum was dominated by protonated molecules $\mathrm{m} / \mathrm{z}, 372.10$ and 332.10 for lornoxicam and piroxicam and the major fragment ions observed in each product spectrum were at $\mathrm{m} / z, 121.10$ and 95.20 respectively. The retention times obtained for lornoxicam and piroxicam were 1.98 and $2.14 \mathrm{~min}$, respectively. Representative chromatograms of an aqueous standard with internal standard mixture, blank plasma and plasma samples spiked with lornoxicam and IS were shown in Fig. 3-5.
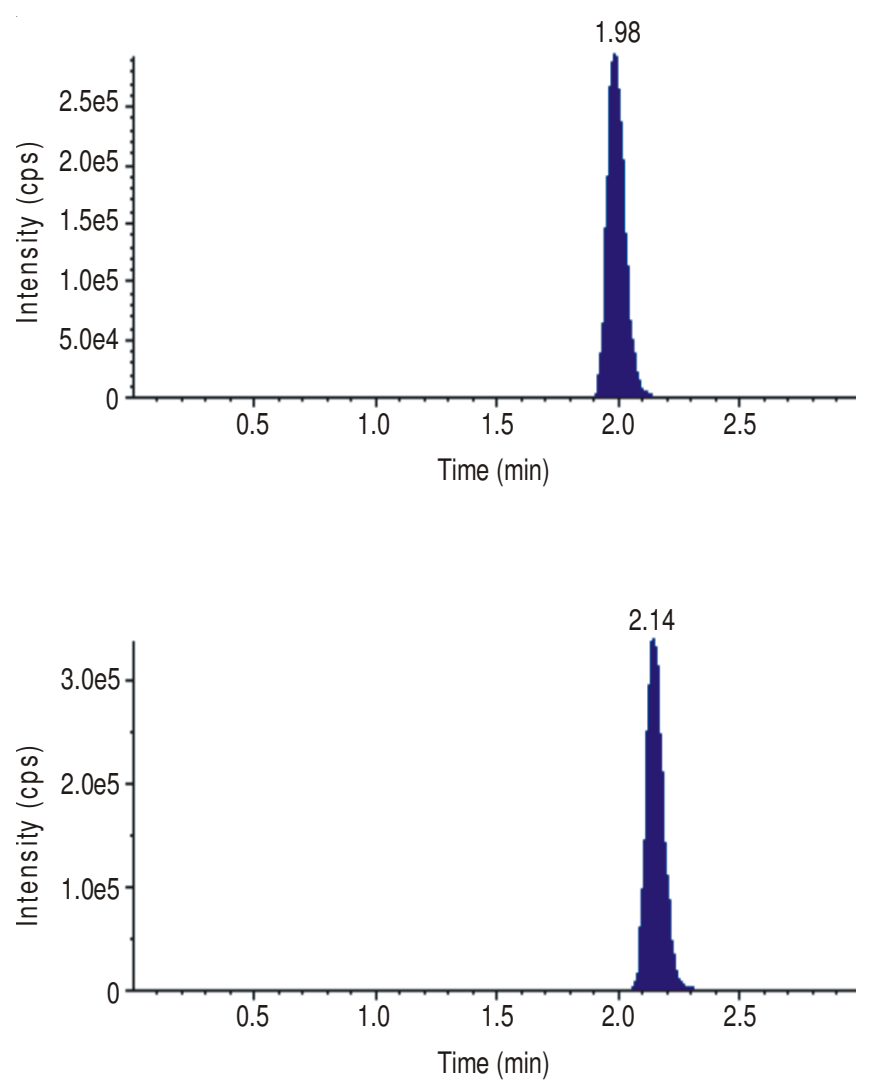

Fig. 3. Representative chromatogram of an aqueous standard and internal standard mixture 
Linearity: The correlation coefficient for lornoxicam over the concentration range of 5.086 to $1518.325 \mathrm{ng} / \mathrm{mL}$ was 0.9991 . The average slope and intercept of regression equations were 0.0001 and 0.0034 , respectively. Linearity was found to be quite satisfactory and reproducible and represented by (Fig. 6).
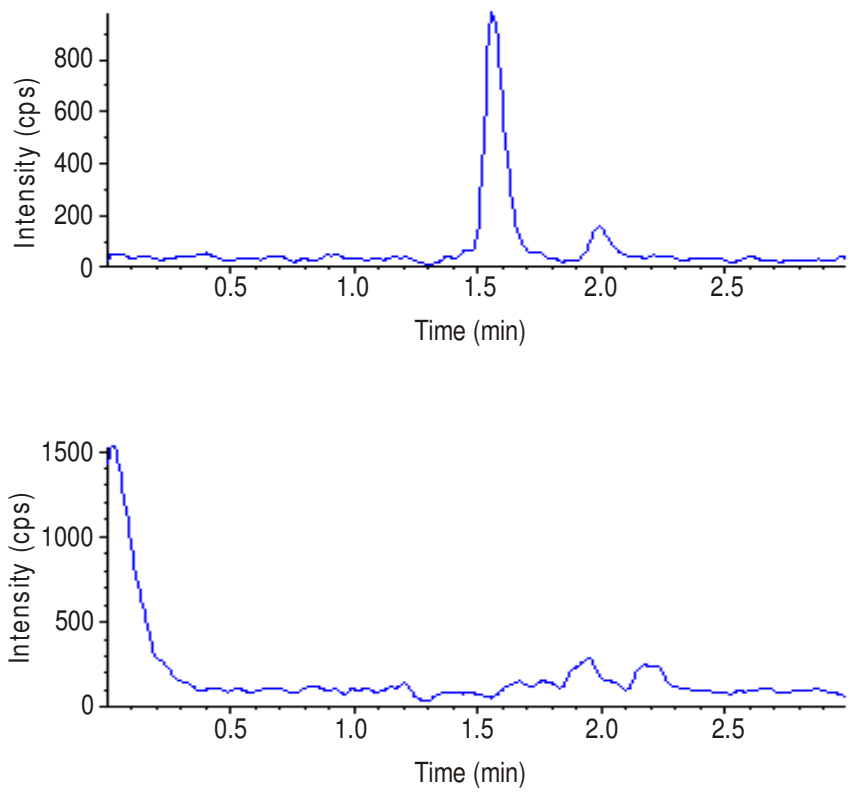

Fig. 4. Representative chromatogram of blank plasma sample
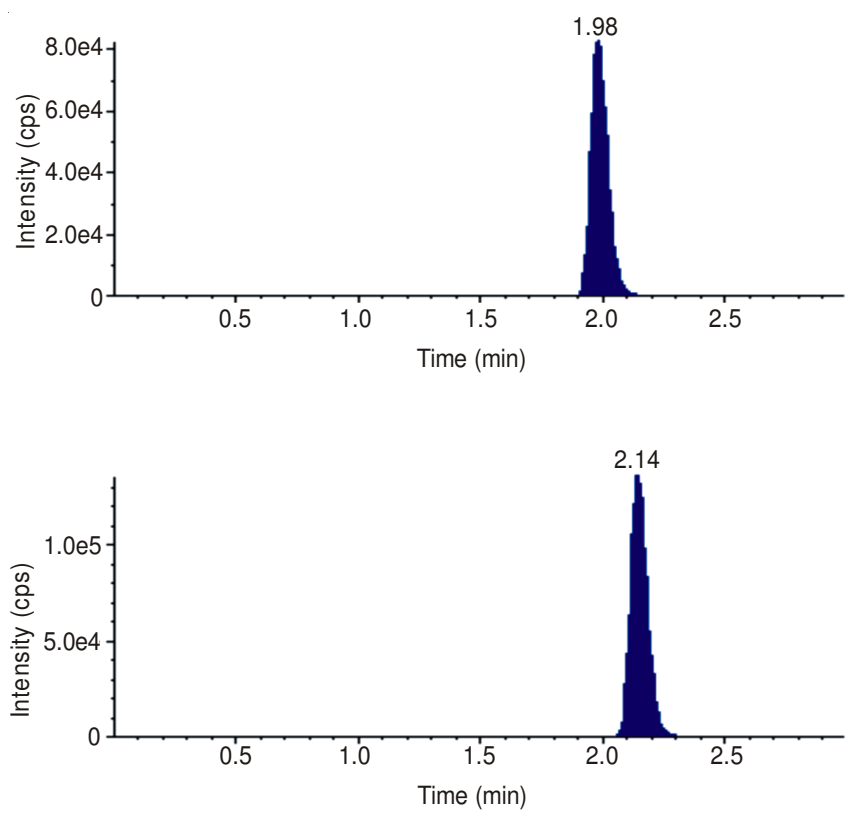

Fig. 5. Representative chromatogram of MQC2 sample of lornoxicam with internal standard

Selectivity: There was no significant interference from endogenous components observed at the mass transitions of lornoxicam and internal standard.

Sensitivity: The lowest limit of reliable quantification for lornoxicam was set at the concentration of the LLOQ, 5.071 $\mathrm{ng} / \mathrm{mL}$ and the results for sencitivity are shown in Table-1. The precision and accuracy for lornoxicam at this concentration was found to be 5.18 and $112.34 \%$, respectively.

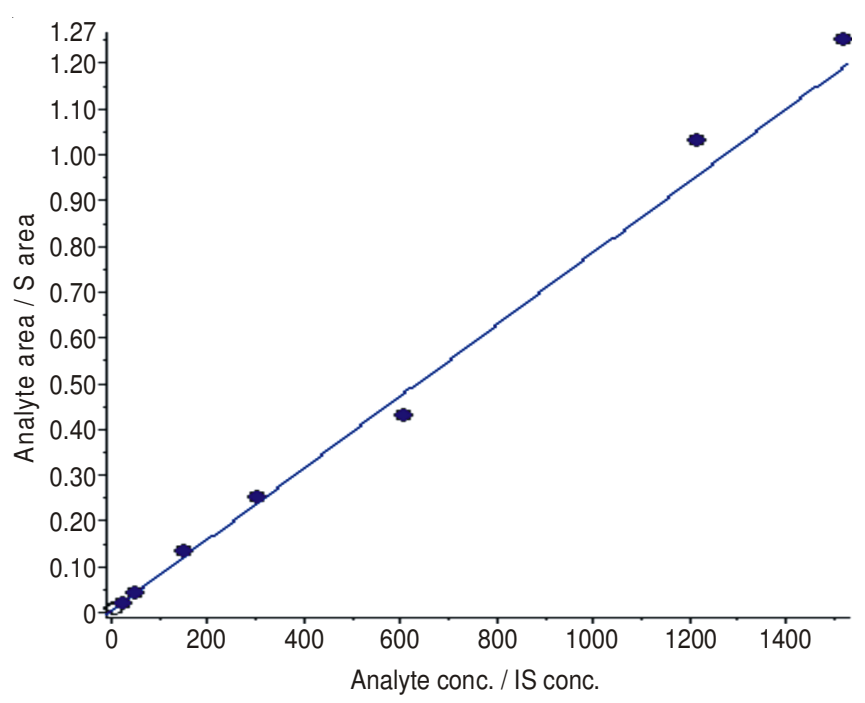

Fig. 6. Representative calibration curve for regression analysis of lornoxicam

\begin{tabular}{cc} 
TABLE 1 \\
WITHIN BATCH PRECISION AND ACCURACY \\
FOR SENSITIVITY OF LORNOXICAM \\
\cline { 2 - 2 } Sample ID & Concentration $(\mathrm{ng} / \mathrm{mL})$ \\
\cline { 2 - 2 } & LLOQ \\
\hline 1 & 5.071 \\
2 & 5.800 \\
3 & 5.491 \\
4 & 5.506 \\
5 & 5.474 \\
6 & 5.671 \\
Mean & 6.240 \\
S.D $(+/-)$ & 5.6970 \\
C.V. $(\%)$ & 0.29488 \\
$\%$ Nominal & 5.18 \\
N & 112.34 \\
\hline
\end{tabular}

Extraction recovery: The percent recoveries of lornoxicam and piroxicam are shown in Tables 2 and 3, respectively. The extraction recoveries determined were found to be $70.98 \%$ with a precision ranging from 0.76 to $7.91 \%$ for lornoxicam and $71.02 \%$ with a precision ranging from 1.18 to $1.78 \%$ for piroxicam. The results are well within the limits.

Accuracy and precision: The intra-day precision and accuracy data and inter day precision and accuracy for QCs are summarized in Tables 4 and 5. The international acceptance criteria were met in each case ${ }^{14}$.

Stability: The processing and storage conditions of clinical samples need to maintain the integrity of a drug or at least keep the variation of pre-analysis as minimal as possible ${ }^{15}$. For this reason, stability studies play an important role in a bio analytical method development. In this study, the stability was assessed by considering different studies such as room temperature stock solution stability, refrigerated stock solution stability, room temperature spiking solution stability, refrigerated spiking solution stability, freeze-thaw, short term stability, bench top stability etc. The results presented in Table-6 shows that lornoxicam is stable under the studied conditions, since in all cases the international acceptance criteria (variation values for area smaller than $15 \%$ ) were met $^{16}$. 
Matrix effect: There is no significant matrix effect was observed in all the eight batches including hemolysis and lipemic (batch no. P040310-253, P050510-287, P050510-288, P240610-309, P240610-313, P050510-290, P070310-254 (lipemic) and P070310-255 (hemolysis)) plasma for lornoxicam at low (LQC) and high (HQC) concentrations. The precision and accuracy for lornoxicam at LQC concentration was found to be 1.13 and $96.29 \%$, respectively and at HQC concentration was found to be 5.17 and $94.93 \%$, respectively and given in Table-7.

TABLE-2

RECOVERY OF LORNOXICAM FROM HUMAN PLASMA

\begin{tabular}{ccc|cc|cc}
\hline & \multicolumn{2}{c|}{ LQC Response } & \multicolumn{2}{c|}{ MQC2 Response } & \multicolumn{2}{c}{ HQC Response } \\
\cline { 2 - 7 } & Extracted QC & Non Extracted QC & Extracted QC & Non Extracted QC & Extracted QC & Non Extracted QC \\
\hline Sample ID & LQC (07-12) & LQC (1-6) & MQC-2 (07-12) & MQC-2(1-6) & HQC (07-12) & HQC (1-6) \\
1 & 13779 & 23423 & 755694 & 1083876 & 1406681 & 1871379 \\
2 & 16174 & 22584 & 735315 & 1071141 & 1402984 & 1846192 \\
3 & 15923 & 23506 & 752772 & 1089191 & 1383853 & 1843838 \\
4 & 17193 & 22874 & 742229 & 1079307 & 1380593 & 1887485 \\
5 & 17286 & 22441 & 748231 & 1093403 & 1413012 & 1858840 \\
6 & 16435 & 22701 & 751970 & 1090403 & 1406291 & 2093833 \\
Mean & 16131.7 & 22921.5 & 747701.8 & 1084553.5 & 1398902.3 & 1900261.2 \\
SD & 1275.25 & 444.70 & 7634.97 & 8269.42 & 13360.81 & 96218.74 \\
CV (\%) & 7.91 & 1.94 & 1.02 & 0.76 & 6.96 & 5.06 \\
N & 6 & 6 & 6 & 68 & 6 \\
\hline Recovery (\%)
\end{tabular}

TABLE-3

RECOVERY OF PIROXICAM FROM HUMAN PLASMA

\begin{tabular}{cccc}
\hline Extracted QC ID & $\begin{array}{c}\text { IS Response in extracted } \\
\text { samples (area) }\end{array}$ & Non-Extracted QC ID & $\begin{array}{c}\text { IS Response in Non-Extracted } \\
\text { Samples (Area) }\end{array}$ \\
\hline MQC2-7 & 1130117 & NON EXTRACTED-MQC-2-1 & 1596096 \\
MQC2-8 & 1090395 & NON EXTRACTED-MQC-2-2 & 1576012 \\
MQC2-9 & 1125548 & NON EXTRACTED-MQC-2-3 & 1583342 \\
MQC2-10 & 1151154 & NON EXTRACTED-MQC-2-4 & 1548819 \\
MQC2-11 & 1130426 & NON EXTRACTED-MQC-2-5 & 1591354 \\
MQC2-12 & 1116161 & NON EXTRACTED-MQC-2-6 & 1599503 \\
Mean & 1123966.8 & Mean & 1582521.0 \\
SD & 20045.88 & SD & 18598.69 \\
CV $(\%)$ & 1.78 & CV (\%) & 1.18 \\
N & 6 & N & 6 \\
\hline Recovery $(\%)$ & & 71.02 & \\
\hline
\end{tabular}

TABLE-4

INTRADAY PRECISION AND ACCURACY FOR LORNOXICAM

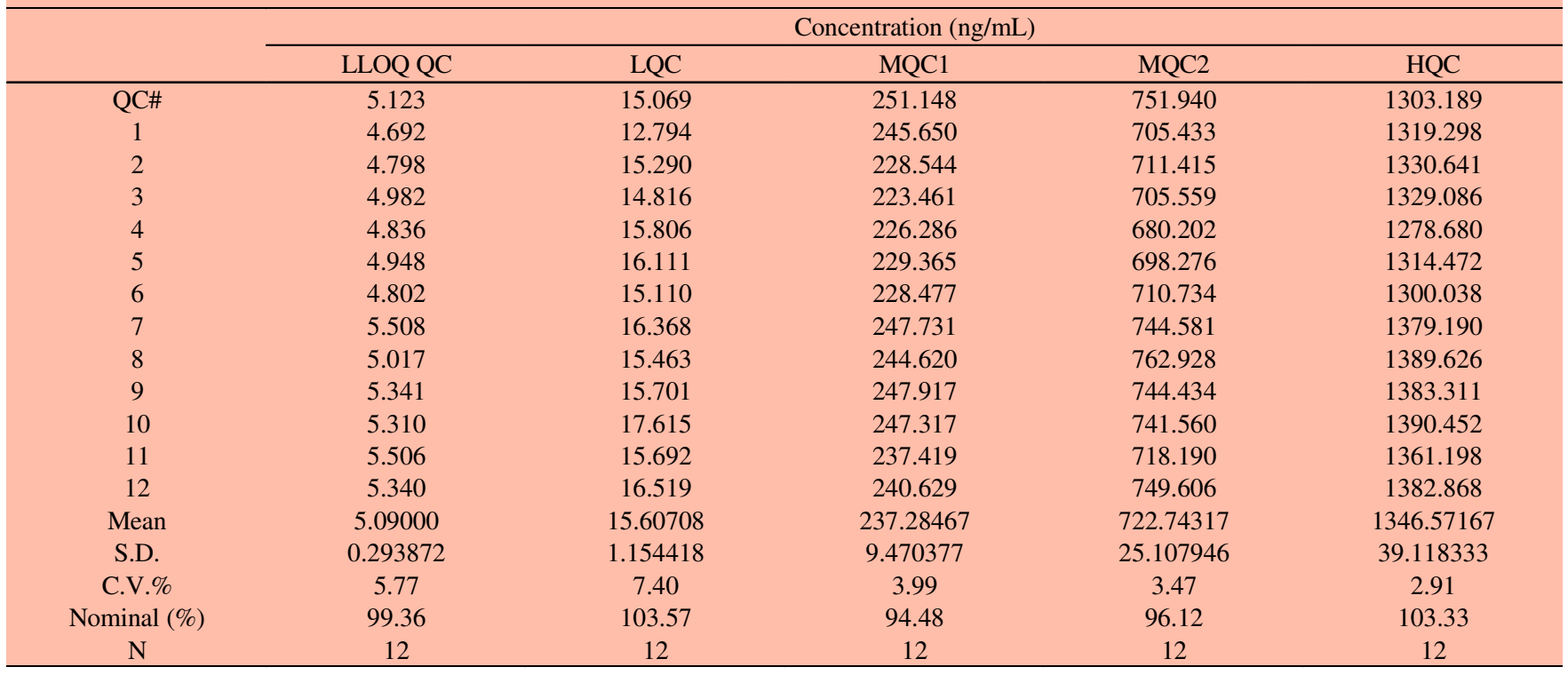




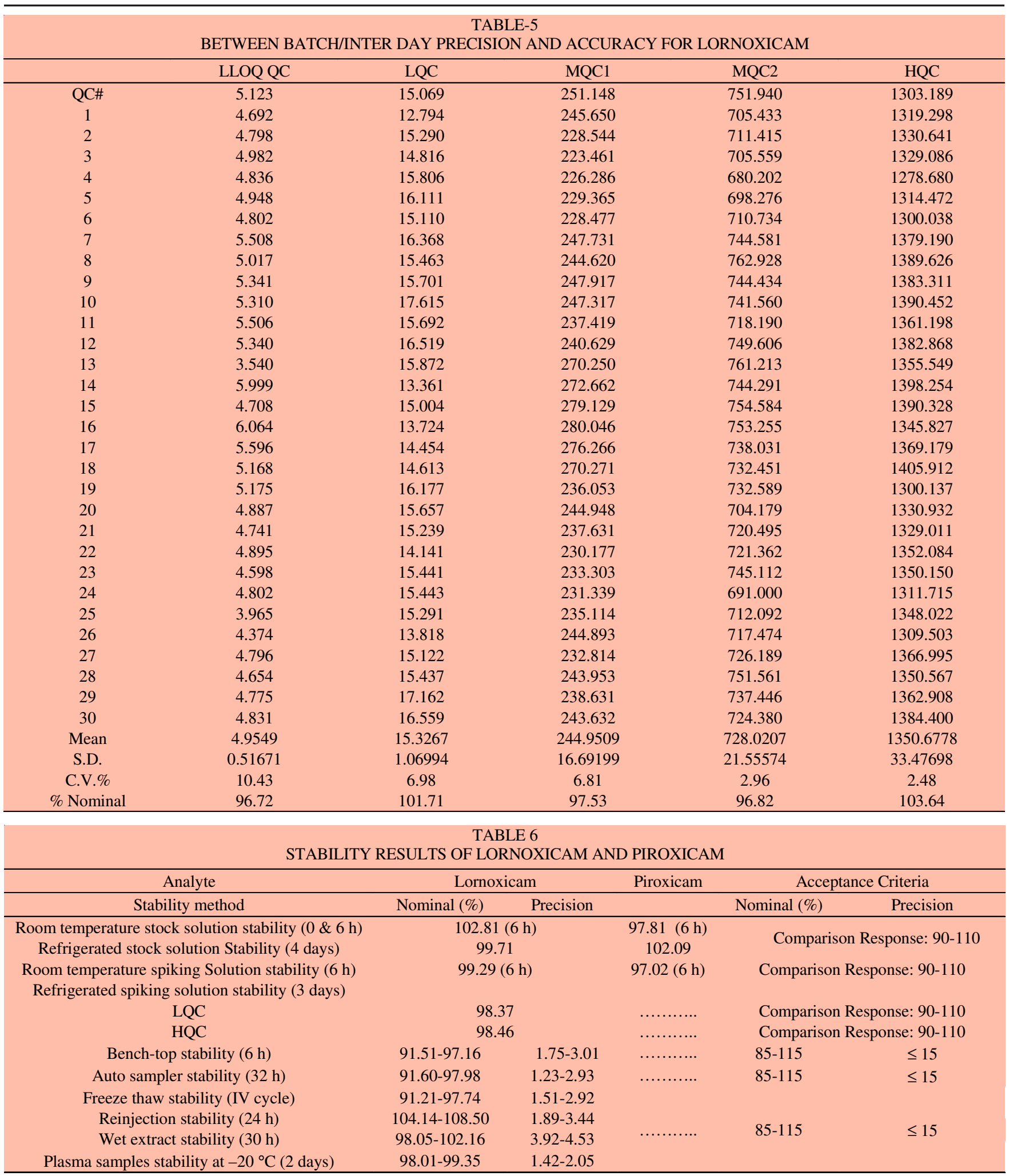

\section{Conclusion}

An alternative HPLC/ESI/MS/MS method for quantification of lornoxicam in human plasma has been successfully developed and validated. A simple and inexpensive liquid liquid extraction procedure and an isocratic chromatography condition using a reversed-phase column provided an assay well suited for real time analysis. The method exhibited excellent performance in terms of selectivity, linearity, accuracy, precision, recovery, stability and matrix effect test. In addition, the reported method has a short analysis run time, an advantage over previously reported methods. Therefore, this method is suitable for therapeutic drug monitoring of lornoxicam.

\section{ACKNOWLEDGEMENTS}

The authors are thankful to Yalamarty Pharmacy College, Tarluwada, Anandapuram, Visakhapatnam, India and Well Quest Research Laboratory, Hyderabad, India for providing necessary facilities during the work. 
TABLE-7

MATRIX EFFECT OF LORNOXICAM

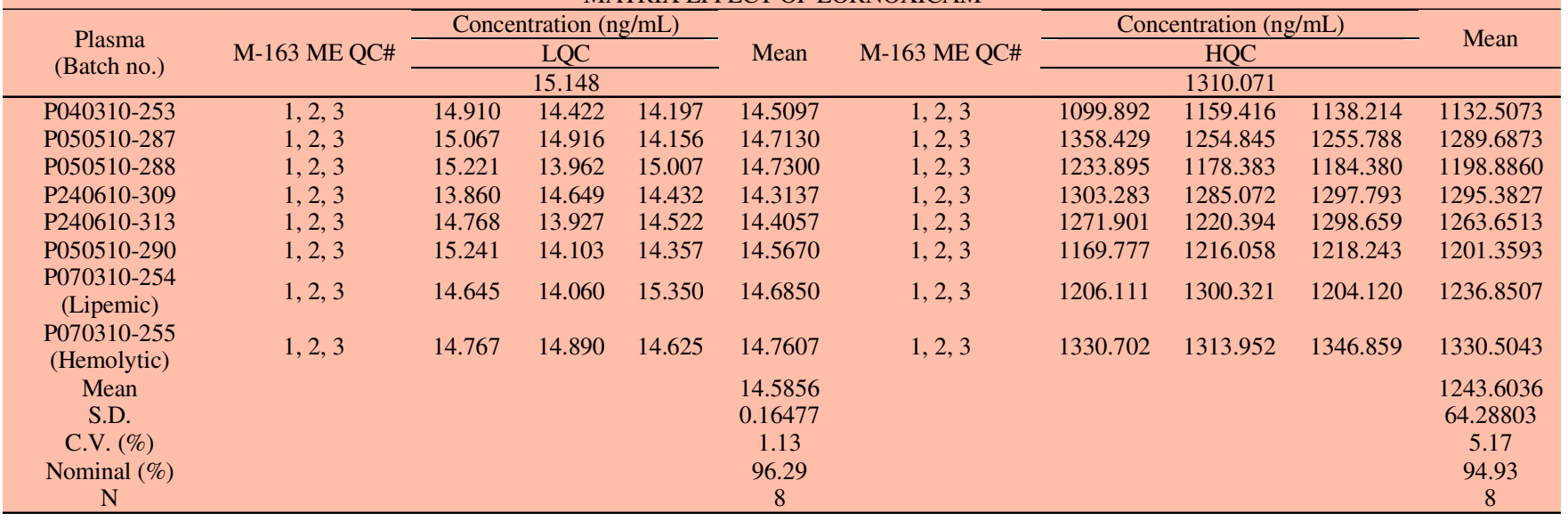

\section{REFERENCES}

1. P. Kumar, S. Shukla, B.B. Subudhi and A.L. Ganure, Int. J. Pharm. Pharm. Sci., 4, 1 (2012).

2. J. Berg, H. Fellier, T. Christoph, J. Grarup and D. Stimmeder, Inflamm. Res., 48, 369 (1999).

3. N. Khisti and S. Bhandari, Asian J. Pharm. Clin. Res., 5, 1 (2012).

4. J.A. Balfour, A. Fitton and L.B. Barradell, Drugs, 51, 639 (1996).

5. M.M. Ghoneim, A.M. Beltagi and A. Radi, Anal. Sci., 18, 183 (2002).

6. I. Cetin, N. Koçak and S. Aycan, C.B.U. J. Sci., 5, 11 (2009).

7. L. Sivasubramanian, K.S. Lakshmi and T. Tintu, Int. J. Pharm. Pharma. Sci, 2, 166 (2010).

8. Y.H. Kim, H.Y. Ji, E.-S. Park, S.-W. Chae and H.S. Lee, Arch. Pharm. Res., 30, 905 (2007).

9. Y.L. Zeng, X.Y. Chen and Y.F. Zhang, Yao Хие Хие Bao, 39, 132 (2004).
10. S. Singh, D.K. Jain and N. Dubey, Int. J. Pharm. Biom. Anal., 5, 57 (2011).

11. W.S. Radhofer and P. Dittrich, J. Chromatogr. B, 707, 151 (1998).

12. V.G. Kulkarni, S.V. Gandhi, P.B. Deshpande and P.H. Chaube, Int. J. Pharm. Pharm. Sci, 3, 225 (2011).

13. A. Nakamura, M.N. Nakashima, M. Wada and K. Nakashima, Bunseki Kagaku, 54, 755 (2005).

14. V.P. Shah, K.K. Midha, S. Dighe, I.J. McGilveray, J.P. Skelly, A. Yacobi, T. Layloff, C.T. Viswanathan, C.E. Cook, R.D. McDowall, K.A. Pittman and S. Spector, J. Pharm. Sci., 81, 309 (1992).

15. E. Mikami, T. Goto, T. Ohno, H. Matsumoto and M. Nishida, J. Pharm. Biomed. Anal., 23, 917 (2000).

16. L. Du, D.G. Musson and A.Q. Wang, J. Pharm. Biomed. Anal., 42, 556 (2006). 